\title{
MULTIPLE ELECTROMAGNETIC EXCITATION OF GIANT DIPOLE PHONONS IN RELATIVISTIC HEAVY ION COLLISIONS
}

\author{
G. BAUR and C.A BERTULANI ${ }^{1}$ \\ Instltut fur Kernphyslk. Kernforschungsanlage Julkch, D-5170 Jultch, Fed Rep Germany
}

Received 24 February 1986

\begin{abstract}
Multıple electromagnetic excitation of giant dipole multıphonon states is studıed in a harmonic vibratıonal model The cross sections are found to be quite large for very heavy nucleı, thus relativistic heavy ion collisions provide a unique tool to study new nuclear states by means of multiphonon excitation and produce new nucles with unusual $N / Z$-ratios
\end{abstract}

The passage of a relativistic heavy ion (RHI) by a nuclear target provides an electromagnetic pulse of a short time duration $t_{\mathrm{coll}} \simeq b / \gamma c$, where $b$ is the impact parameter and $\gamma=\left[1-(v / c)^{2}\right]^{-1 / 2}$. In such a collision virtual photons of energy up to

$E_{\gamma}^{\mathrm{max}}=\hbar / \tau_{\mathrm{coll}} \simeq(\hbar c / b) \gamma$

are present [1]. Such photons can be sufficiently energetic to excite the grant dipole resonance (GDR) (and other high-lying multipole resonances) in RHI collisions. Such excitations have been observed [2] through the subsequent particle decay of the GDR (in the target as well as in the projectule). The strength of the electromagnetic multipole excitation of order E $\lambda$ can be measured [3] in terms of the strength parameter

$\chi_{1 \rightarrow \mathrm{f}}^{(\lambda)}=Z e\left\langle\mathrm{f}|M(\mathrm{E} \lambda)|_{1}\right\rangle / \hbar v b^{\lambda}$,

where $Z$ is the charge number of the projectile and $\langle f|M(\mathrm{E} \lambda)| 1\rangle$ the electromagnetic multipole moment of the corresponding transition in the target nucleus.

In the case of non-relativistic Coulomb excitation where $v \ll c$, the strength parameter $\chi_{1 \rightarrow \mathrm{f}}^{(\lambda)}$ can be quite large, providing the possibility of multıple Coulomb excitation, especially by means of heavy ions. For RHI on the other hand, this strength parameter will not exceed the value of one, not even for very hugh $Z$.

\footnotetext{
On leave of absence from Federal University of Rio de Janeıro. Rio de Janeıro, Brazıl
}

The multipole matrix element $\left\langle\mathrm{f}|M(\mathrm{E} \lambda)|_{1}\right\rangle$ is of the order of $e R^{\lambda}$, where $R$ denotes the nuclear radius, therefore $\chi_{1 \rightarrow f}^{(\lambda)} \simeq Z\left(e^{2} / \hbar c\right)(R / b)^{\lambda}$ which will be less than one $\left(Z \vec{e}^{2} / \hbar c=Z \alpha<1\right)$, since the impact parameter $b$ has to be chosen larger than $R$ in order to obtain a pure electromagnetic excitation process. Therefore the first-order semiclassical theory [3] and its quantal versions $[4,5]$, like the PWBA (modified by a cylindrical hole cut-off), will generally account well for electromagnetic excitation effects in RHI coll-sions.

It has been observed in ref. [4] that the absolute values of the semiclassical excitation amplitudes $a_{\mathrm{f}}$ for the excitation of the GDR in very heavy nucles by very heavy relativistic nucles (like in $U-U$ collisions) will approach unit magnitude. In such a situation the assumed first-order perturbation theory can no longer be accurate and higher-order effects must be taken into account.

Instead of giving a general theory of multiple excitation in RHI collisions (by means of e.g. semuclassical coupled channels or Glauber theones) we consider the problem of multiple excitation of a pure harmonic oscillator. We note that this problem can be solved (to all orders) in closed form $[6,7]$. The multuple excitation amplitude can be directly expressed in terms of the first-order amplitudes, which have already been calculated $[3,4]$.

Since it cannot be studied with non-relativistic heavy ions due to the adiabaticity condition, the ex- 
citation of two (and more) GDR phonons is of spec1al interest. To our knowledge, nothing is known experimentally about such states (they would e.g. not be seen in photoabsorption, since a two-photon absorption mechanism would be required to excite such states; in atomic physics such kinds of excitations are of course widely achieved nowadays with lasers). In such a situation we therefore try to use the simplest possible model, the collective oscillation of all neutrons against all protons in terms of (undamped) harmonic vibrations. The usual GDR is a one-photon state of angular momentum 1 (assuming a spin zero nuclear ground state $|0\rangle)$

$|1,1 \mu\rangle=a_{\mu}^{\dagger}|0\rangle$,

where $a_{\mu}^{\dagger}$ denotes the creation operator for a GDR photon with angular momentum projection $\mu$. The operators $a_{\mu}^{\dagger}$ and $a_{\mu}$ satisfy the usual boson commutation relations. The two phonon states $|2, I M\rangle$, coupled to good angular momentum $I, M$ with $I=0$ and 2 , are given explicitly by (see e.g. ref. [7])

$|2, I M\rangle=(1 / \sqrt{2}) \sum_{\mu \mu^{\prime}}\left\langle 1 \mu 1 \mu^{\prime} \mid I M\right\rangle a_{\mu}^{\dagger} a_{\mu}^{\dagger},|0\rangle$.

The dipole transition matrix elements are obtained from the TRK sum rule

$$
\int \sigma_{\gamma}^{\mathrm{E} 1}\left(E_{\gamma}\right) \mathrm{d} E_{\gamma} \simeq 60 N Z / A \mathrm{MeV} \mathrm{mb},
$$

and the experimentally known energy position of the GDR

$E_{\mathrm{GDR}}=80 \mathrm{MeV} / A^{1 / 3}$,

assuming that the GDR exhausts the sum rule, eq.

(5a). This is a reasonable assumption for our purposes. With these numbers we calculate $[3,4,8]$ the first-order excitation amplitude $a_{0 \rightarrow 1}^{m}$, where $m$ denotes the angular-momentum transfer in the beam direction. One obtains for the excitation of a nucleus $\left(N_{2}, Z_{2}\right)$ by a nucleus with charge $Z_{1}$

$\left|a_{0 \rightarrow 1}^{m=0}\right|=0.41 \alpha\left[Z_{1}\left(N_{2} Z_{2}\right)^{1 / 2} / A_{2}^{2 / 3}\right](c / \gamma v)^{2} K_{0}(\xi)$,

and

$$
\begin{aligned}
& \mid \begin{array}{l}
\mid a_{0 \rightarrow 1}^{m}= \pm 1 \\
0 \rightarrow 1
\end{array} \\
& \quad=0.29 \alpha\left[Z_{1}\left(N_{2} Z_{2}\right)^{1 / 2} / A_{2}^{2 / 3}\right](c / v)^{2}(1 / \gamma) K_{1}(\xi),
\end{aligned}
$$

where the adiabaticity parameter $\xi$ is given by

$\xi=E_{\gamma} b / \hbar v \gamma$.

The total excitation probability of the one-phonon GDR is given, in first-order perturbation theory, by

$P_{1}^{(\text {first order })}=\sum_{m=-1}^{1}\left|a_{0 \rightarrow 1}^{m}\right|^{2} \equiv|\chi(b)|^{2}$,

where we have introduced a parameter $|\chi(b)|^{2}$. In the exact theory of multiple Coulomb excitation of a harmonic oscillator $[6,7]$ one obtains a Poisson distribution for the excitation probability $P_{N}$ of an $N$-phonon state

$P_{N}=(1 / N !)|\chi(b)|^{2 N} \exp \left[-|\chi(b)|^{2}\right]$,

where $|\chi(b)|^{2}$ is given in eq. (7). In refs. [6,7] the theory was developed for nonrelativistic Coulomb excitation, the same arguments apply also to the relatıvistic case.

From eq. (6) it is seen directly that the largest excitation amplitudes are obtained the larger the projectile and the target area. Therefore we study the example of ${ }^{238} \mathrm{U}+{ }^{238} \mathrm{U}$ collisions. In fig. 1 the impact parameter dependent excitation probabilities $P_{N}$ given by eq. (8) using eqs. (7) and (6) are shown. The probabilities for $N=1$ decrease essentıally like $1 / b^{2}$ untıl

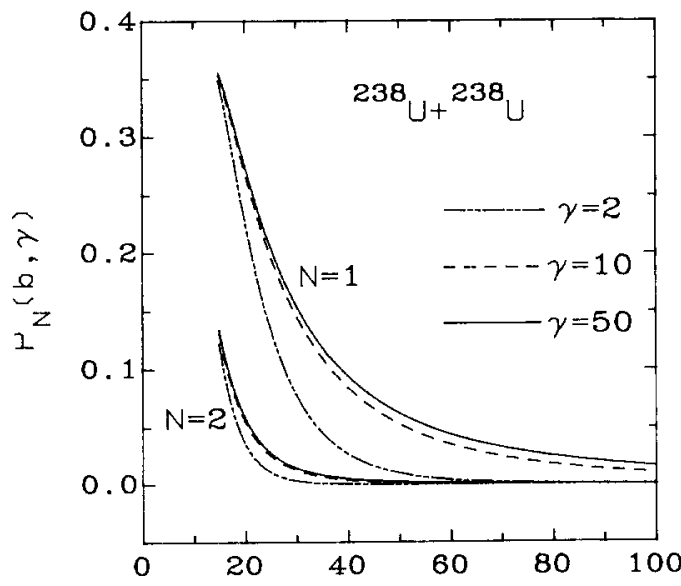

Impact parameter $(f m)$

Fig. 1 The excitation probability $P_{N}$ of $N$-phonon GDR states is shown as a function of the impact parameter $b$ for ${ }^{238} U+{ }^{238} U$ RHI collisions. 


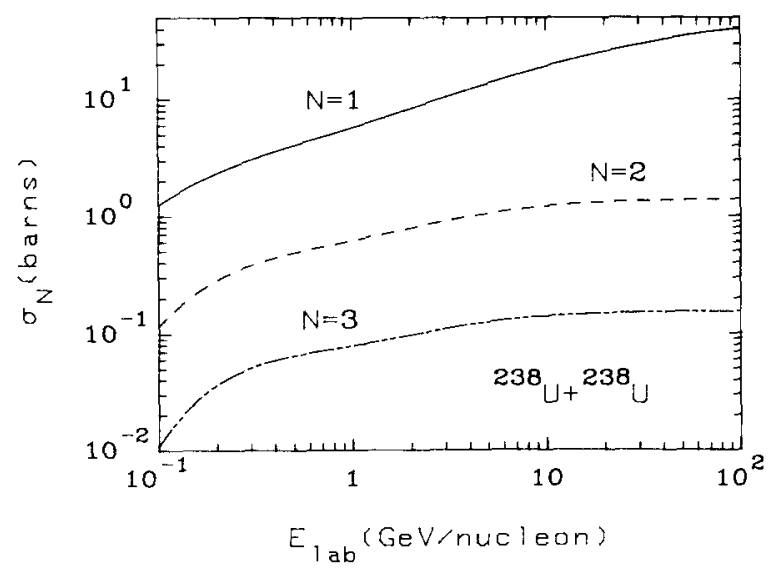

Fig 2 The total cross section $\sigma_{N}$ for the excitation of $N$ phonon GDR states in ${ }^{238} \mathrm{U}-238 \mathrm{U}$ RHI collisions as a function of the incident energy $E_{\text {lab }}(\mathrm{GeV} /$ nucleon)

the adiabatic cut-off [see eq. (1)] is reached. The probabilities for $N>1$ decrease faster $\left(\sim 1 / b^{2 N}\right)$ and are of smaller magnitude, as it is expected. However, their values are still quite appreciable. In fig. 2 the total cross sections $\sigma_{N}$ for the excitation of $N$-phonon GDR states

$\sigma_{N}=2 \pi \int_{R_{1}+R_{2}}^{\infty} b \mathrm{~d} b P_{N}(b)$

are given. The minimum impact parameter is given by $R_{1}+R_{2}$, where $R_{l}=1.2 A_{l}^{1 / 3}$ fm $(i=1,2)$. It can be seen that the cross section for $N=1$ is larger than the geometrical cross section for $E_{\mathrm{lab}} \gtrsim 2 \mathrm{GeV} /$ nucleon. It increases logarithmically with $\gamma$. The cross sections for $N \geqslant 2$ are smaller, however still large as compared to nuclear fragmentation cross sections. They tend to a constant value as $\gamma \rightarrow \infty$, as can be directly shown from the behaviour of $\left|a_{0 \rightarrow 1}(b)\right|^{2}$ [eqs (6), (8)].

The one-phonon GDR excitations in RHI collisions have been observed experımentally (see e.g. ref. [2]) with the theoretically expected large cross sections The experimental signature of that process was the observation of fragments which have lost one or two nucleons (mainly neutrons) by the decay of the GDR. Since this particle decay is very fast (the GDR is a strongly damped collective motion) it may be asked whether a two- or more-phonon excitation is possible at all. The decay tıme of GDR corresponding to a width of $\Gamma=5 \mathrm{MeV}$ is given by

$\tau_{\text {decay }}=\hbar / \Gamma \simeq 10^{-22} \mathrm{~s}$.

This time has to be compared to the passage time $t_{\text {coll }} \simeq b / \gamma c \sim(5 / \gamma) \times 10^{-23} \mathrm{~s}$ for $b=15 \mathrm{fm}$. Thus we will have $\tau_{\text {decay }}>\tau_{\text {coll }}$ even for moderately high values of $\gamma$ and multiple excitation will be possible in principle. Nothing is known about the widths of the higher $(N \geqslant 2)$ phonon states, if the widths of these states would become larger than the level spacing $E_{\mathrm{GDR}}$ [see eq. (5b), $13 \mathrm{MeV}$ for $A=238$ ] then the observation of such states would become difficult. The experimental detection of such two- (or more-) phonon GDR states is a challenge, the signature could be fragments where quite a few neutrons have been removed in this grant oscillation of neutrons against protons ("cold fragmentation", see Kienle [9]). This electromagnetic excitation process can therefore be a promising method to produce beams of new nucle1 with unusual $N / Z$-ratios, this can lead to further interestıng studies.

The two-phonon multıplet contains angular momentum states $I=0$ and 2 . These states are explicitly given by [see eq (4)]

$$
\begin{aligned}
& |2,00\rangle=(1 / \sqrt{6})\left(2 a_{1}^{\dagger} a_{-1}^{\dagger}-a_{0}^{\dagger} a_{0}^{\dagger}\right)|0\rangle \\
& =\sqrt{2 / 3}|1,0,1\rangle-(1 / \sqrt{3})|0,2,0\rangle, \\
& |2,20\rangle=(1 / \sqrt{3})\left(a_{0}^{\dagger} a_{0}^{\dagger}+a_{1}^{\dagger} a_{-1}^{\dagger}\right)|0\rangle \\
& =\sqrt{2 / 3}|0,2,0\rangle+(1 / \sqrt{3})|1,0,1\rangle, \\
& |2,21\rangle=a_{1}^{\dagger} a_{0}^{\dagger}|0\rangle=|0,1,1\rangle, \\
& |2,22\rangle=(1 / \sqrt{2}) a_{1}^{\dagger} a_{1}^{\dagger}|0\rangle=|0,0,2\rangle,
\end{aligned}
$$

and similarly for $M=-1$ and -2 . Here we have introduced the uncoupled normalized states $\left|n_{-1}, n_{0}, n_{1}\right\rangle$, where $n_{\mu}$ denotes the number of phonons with angular momentum projection $\mu$. For $\gamma \gg 1$, the $m= \pm 1$ excitation amplitude completely dominates over the $m=0$ excitation (see e.g. ref. [1]). Neglecting $\left|a_{0 \rightarrow 1}^{m=0}\right|$ [eq. (6a)] as compared to $\left|a_{0 \rightarrow 1}^{m= \pm 1}\right| \equiv x$ [eq. (6b)] we obtain a simple and realistic result for the excitation of the angular momentum states. In terms of $x$ we obtain for the excitation probability $P_{n_{-1}} n_{0} n_{1}$ of the uncoupled states $\left|n_{-1}, n_{0}, n_{1}\right\rangle$ (see ref. [7], p. 199ff) 
$P_{101}=x^{4} \exp \left(-2 x^{2}\right)$

$P_{002}=P_{200}=\frac{1}{2} x^{4} \exp \left(-2 x^{2}\right)$.

The other combinations give only a negligible contribution. From eq. (11) we obtain for the excitation probability $P_{2, I M}$ of the angular momentum coupled states $|2, I M\rangle$

$P_{2,22}=P_{2,2-2}=\frac{1}{2} x^{4} \exp \left(-2 x^{2}\right)$,

$P_{2,21}=P_{2,2-1}=0$,

$P_{2,20}=\frac{1}{3} x^{4} \exp \left(-2 x^{2}\right)$,

$P_{2,00}=\frac{2}{3} x^{4} \exp \left(-2 x^{2}\right)$.

The total $N=2$ excitation probability adds of course up to the value $P_{N=2}$ which has already been obtained above [eq. (8) with $2 x^{2}=|\chi(b)|^{2}$ ]. The ratio of exc1tation of $0^{+}$and $2^{+}$states is $1: 2$.

In conclusion, the use of RHI offers a unique method to study multiphonon GDR states, nuclear collective states very high up in the continuum hitherto unexplored. The multinucleon decay of these states offers a new method to produce nucles with very unusual $N / Z$-ratios. Our simple model should give a farrly reliable estimate of the cross sections.
They are found to be quite appreciable. However it remains to be seen in new experiments with RHI what the properties of these new and exotic nuclear states really are, especially what their particle decay widths are, their anharmonicities, and how the strong damping of the giant dipole oscllation will affect these states.

This work was partly supported by Deutscher Akademischer Austauschdienst/CAPES.

\section{References}

[1] J D. Jackson, Classical electrodynamics (Wiley, New York, 1975).

[2] D.L. Olson, B L. Berman, D.E. Greiner, H.H. Heckman, P.J. Lindstrom, G D. Westfall and H.J. Crawford, Phys. Rev. C24 (1981) 1529, M.T. Mercier, J.C Hill, F.K. Wohn and A.R. Smith, Phys. Rev. Lett. 52 (1984) 898.

[3] A Winther and K. Alder, Nucl Phys. A319 (1979) 518.

[4] C.A. Bertulanı and G Baur, Nucl. Phys. A442 (1985) 739

[5] C.A. Bertulanı and G Baur, Phys. Rev. C 33 (1986) 910.

[6] K. Alder and A. Winther, Mat Fys. Medd. Dan. Vid. Selsk. 32, No. 8 (1960).

[7] K. Alder and A Winther, Electromagnetic excitation (North-Holland, Amsterdam, 1975).

[8] C.A. Bertulanı and G. Baur, submitted to Nucl. Phys. A.

[9] P. Kienle, Nucl Phys. A447 (1985) 419c. 